G. S. B. Wijethunga ${ }^{1}$, S. D. Amarasinghe ${ }^{1}$, T. M. R. Perera ${ }^{1,2}$, P. I. Jayasekera ${ }^{3}$, R. Sathanandaraja ${ }^{1}$, N. S. Chandrasiri ${ }^{1}$ ${ }^{1}$ Colombo South Teaching Hospital, Kalubowila, Sri Lanka;

${ }^{2}$ University of Sri Jayewardenepura, Nugegoda, Sri Lanka; and

${ }^{3}$ Medical Research Institute, Colombo 08, Sri Lanka

Aim: Candida species causing neonatal candidaemia show a wide geographical variation and are usually healthcare acquired. Awareness of prevalent Candida species and knowledge of possible risk factors allows timely commencement of antifungal prophylaxis, directs appropriate empirical antifungal therapy and helps to prevent occurrence. This study aims to evaluate the Candida species distribution and the impact on preventive measures.

Method: This was carried out as a prospective descriptive study in a Special Care Baby Unit (with a capacity of 16 cots with ventilation facilities for 8 newborns) attached to a tertiary care hospital in Colombo, Sri Lanka from January 2015 to December 2020. Identification and antifungal sensitivities were done according to standard protocols. Impact of preventive measures implemented to prevent nosocomial transmission of Candida species was reassessed.

Result: A total of 27 candidaemia episodes (C. parapsilosis $61.54 \%$, C. albicans $15.38 \%$, C. guilliermondii $15.38 \%$ and $7.7 \%$ non-albicans Candida) were detected during the study period. The overall incidence was 1.04 per 100 SCBU admissions. A drop in antibiotic consumption was noted with antibiotic stewardship implementation.

Discussion: Neonatal candidaemia was predominantly caused by non-albicans Candida species. Implementation of antibiotic stewardship programme and strengthening of infection control activities markedly reduced the incidence of neonatal candidaemia.

\section{THE UTILITY OF THE SYPHILIS ENZYME IMMUNOASSAY IGM IN THE DIAGNOSIS OF CONGENITAL SYPHILIS}

Stephanie Hendry $^{1}$, Madeleine Kelly ${ }^{2}$, Robert Norton ${ }^{1}$ ${ }^{1}$ Pathology Queensland, Townsville Hospital, Townsville, Qld, Australia; and ${ }^{2}$ School of Medicine, James Cook University, Townsville, Qld, Australia

Background: Congenital syphilis has increasing incidence in Australia, with significant sequelae. Effective screening tests are available during pregnancy; however, they rely on adequate antenatal care. Congenital syphilis can be a challenging diagnosis to make, with a variety of clinical and laboratory factors to consider. The syphilis IgM routinely ordered as part of the diagnostic process.

Aim: To assess the utility of the syphilis EIA IgM test in the diagnosis and treatment of congenital syphilis.

Methods: Laboratory and clinical records of mothers and newborns who were syphilis EIA IgM reactive were reviewed over a ten-year period.

Results: There were 11 syphilis IgM reactive results from 371 tests performed $(3 \%)$. Nine of these cases were reviewed in detail. The IgM result was never a key factor in establishing a diagnosis or instigating treatment in an infant.

Discussion: Use of maternal serology and treatment history are better tools to identify those neonates at risk of congenital syphilis, rather than the EIA syphilis IgM result. In cases when antenatal maternal serology is unavailable, PCR can provide valuable a timely insight in at risk neonates. This study highlights the low value of the syphilis IgM in the diagnostic management of congenital syphilis.

\section{SPINAL TOXOPLASMOSIS IN A YOUNG MAN WITH UNDIAGNOSED HIV INFECTION}

Annaleise R. Howard-Jones ${ }^{1,2}$, Zoe Jennings ${ }^{2,3}$, Stuart Adams $^{1,2}$, Harsha Samarasekara ${ }^{1,2}$

${ }^{1}$ NSW Health Pathology (Nepean), Nepean Hospital, Kingswood, NSW, Australia; ${ }^{2}$ Faculty of Medicine \& Health, University of Sydney, Camperdown, NSW, Australia; and ${ }^{3}$ Department of Microbiology \& Infectious Diseases, Nepean Hospital, Kingswood, NSW, Australia

Spinal cord lesions are potentially devastating conditions leading to reduced mobility, loss of functional independence, and even ventilator dependence, dictated by the level of injury. Tissue diagnosis is paramount to differentiate between primary brain tumours such as ependymomas and glioblastomas, infarction, infection and demyelination.

We present the case of a 30-year-old gentleman with subacute neck pain, nausea and evolving right upper limb weakness and paraesthesiae. MRI-spine demonstrated an intramedullary cervical spine mass with associated oedema, thought to represent an ependymoma. Open tissue biopsy revealed numerous bradyzoites on haematoxylin \& eosin staining, subsequently confirmed by directed polymerase chain reaction testing as Toxoplasma gondii. Detailed immunological work-up led to a diagnosis of HIV infection, with detection of HIV antibodies and HIV-1 p24 antigen, an HIV-1 viraemia ( $\log 6.33$ copies $/ \mathrm{mL})$ and low $\mathrm{CD} 4$ count $(16$ cells $/ \mathrm{mL})$.

Toxoplasmosis is the most common central nervous system infection in HIV patients not receiving appropriate prophylaxis. ${ }^{1}$ It may occur in up to $30 \%$ of such patients who are seropositive for Toxoplasma gondii, ${ }^{2}$ typically manifesting when CD4+ counts fall below 100 cells $/ \mathrm{mm}^{3}{ }^{3}$. This case highlights the importance of close collaboration between histopathology, microbiology and infectious diseases specialists in diagnosis of a spinal cord infection with associated HIV infection.

References

1. Porter SB, Sande MA. Toxoplasmosis of the central nervous system in the acquired immunodeficiency syndrome. $\mathrm{N} \mathrm{Engl} \mathrm{J}$ Med 1992; 327: 1643-8.

2. Grant IH, Gold JW, Rosenblum M, et al. Toxoplasma gondii serology in HIV-infected patients: the development of central nervous system toxoplasmosis in AIDS. AIDS 1990; 4: 519-21.

3. Kaplan JE, Benson C, Holmes KK, et al. Guidelines for prevention and treatment of opportunistic infections in HIVinfected adults and adolescents. MMWR Recomm Rep 2009; 58: $1-207$.

\section{EXPRESSION OF GPI-ANCHORED SARS-COV-2 RECEPTOR BINDING DOMAIN IN MAMMALIAN CELLS FOR SEROLOGICAL USE}

M. L. Kernich ${ }^{1,2}$, P. J. Adamson ${ }^{2}$, J. J. Wang ${ }^{3}$, L. P. Kris ${ }^{3}$, B. L. Armour ${ }^{3}$, D. Beroukas ${ }^{2}$, G. Best ${ }^{3}$, J. M. Carr ${ }^{3}$, T. P. Gordon ${ }^{2,3,4}$, D. L. Gordon ${ }^{1,2,3}$

${ }^{1}$ Microbiology and Infectious Diseases, Flinders Medical Centre, SA, Australia; ${ }^{2}$ SA Pathology, SA, Australia; ${ }^{3}$ College 
of Medicine and Public Health, Flinders University, SA, Australia; and ${ }^{4}$ Clinical Immunology, Flinders Medical Centre, SA, Australia

Introduction: The gold standard for diagnosing SARS-CoV-2 infection remains RT-PCR; however serological testing has an important role in epidemiology and assessment of vaccine responses. We expressed the receptor binding domain (RBD) of the spike (S) protein as a glycosylphosphatidylinositol (GPI)anchored protein on $\mathrm{CHO}$ cells transfected with a generated construct and determined binding of RBD specific antibodies by immunofluorescence (IF) and flow cytometry (FACS) assays.

Methods and results: IF and FACS demonstrated cell surface RBD expression using anti-S RBD $\mathrm{mAb}$ and macaque immune sera. GPI anchored expression was confirmed through loss of positive cells on FACS post GPI anchor cleavage with phosphoinositide phospholipase C (PI-PLC). Binding of antibodies from COVID-19 convalescent sera to RBD- and mock-transfectants was performed by FACS. Positive results on serial dilutions of patient sera down to 1:3200 were observed and compared to in-house RBD ELISA which lost positivity at a 1:800 dilution. Soluble recombinant ACE2 bound to RBDCHOs and binding was inhibited by COVID-19 convalescent sera indicating the RBD was expressed in a functional conformation and that neutralising antibodies were detected.

Conclusion: Mammalian cells expressing GPI-anchored RBD can successfully be used to detect previous SARS-CoV-2 infection and likely indicate the presence of neutralising antibodies in COVID-19 survivors.

\section{IMPACT OF A DIAGNOSTIC STEWARDSHIP INITIATIVE OF BACTERIAL 16s rRNA SEQUENCING REFERRALS FROM A REGIONAL LABORATORY}

Daniel Lennon, Rob George, Hemalatha Varadhan

New South Wales Health Pathology - Hunter, John Hunter

Hospital, New Lambton Heights, NSW, Australia

Introduction: 16s rRNA PCR (16s) has emerged as a useful tool for the identification of bacterial pathogens in clinical samples1. Due to its increased cost and limited availability, this technology is also a suitable target for diagnostic stewardship initiatives.

Aim: To assess a diagnostic stewardship initiative impact on key laboratory quality indicators for specimen referrals for $16 \mathrm{~s}$ PCR from the John Hunter Hospital Laboratory.

Methods: From 01/07/2019 to 31/12/2019, all referred specimens for 16 s received by our laboratory were held for review by a Microbiologist (or Registrar). Key laboratory quality indicators were collected and compared to a retrospective audit of $16 \mathrm{~s}$ referrals from the preceding 6 months.

Results: 192 referrals were examined, with 77 referrals made in the pre-intervention phase and 115 in the post-intervention phase. 9 referrals were cancelled in the post-intervention phase. The cost per positive 16 s was $\$ 580.04$ in the pre-intervention period and $\$ 375.40$ in the post intervention period. The average turn-around time was 14.61 days in the pre-intervention period, and 13.47 days in the post-intervention period, with no significant difference between the two periods $(p=0.122)$.

Conclusion: Diagnostic stewardship is an economical endeavor for laboratories without increase in turnaround time.

\section{Reference}

1. Janda JM, Abbott SL. 16S rRNA gene sequencing for bacterial identification in the diagnostic laboratory: pluses, perils, and pitfalls. J Clin Microbiol 2007; 45: 2761-4.

\section{FUNGAL LIVER ABSCESSES IN AN IMMUNOCOMPETENT PATIENT POST CHOLECYSTECTOMY - A CASE REPORT}

\section{Laura M. Luttrell}

Department of General Surgery, Bunbury Regional Hospital, Bunbury, WA, Australia

Candida albicans is known to colonise the biliary tract but rarely causes invasive candidiasis post gastrointestinal or hepatobiliary surgery in the absence of host immunosuppression. This case study reports a 33-year-old immunocompetent female with a past surgical history of sleeve gastrectomy, omega loop (mini) gastric bypass and gastric ulcer repair. Two weeks post laparoscopic cholecystectomy and intraoperative cholangiogram (IOC) the patient presented with right upper quadrant abdominal pain, rigors, nausea, vomiting, fevers $(39.5$ degrees) and tachycardia. Liver function tests were mildly deranged, the white cell count was $10 \times 10^{9} / \mathrm{L}$ and the C-reactive protein was elevated at $327 \mathrm{mg} / \mathrm{L}$. Computed tomography (CT) revealed multiple right lobe liver abscesses and mild splenomegaly. The patient underwent ultrasound guided drainage and Candida albicans was subsequently grown from the aspirate sample. After a 4-week course of oral fluconazole, a repeat CT abdomen and pelvis showed good resolution with 3-4 remaining small liver collections. An abdominal ultrasound 8-weeks post initial presentation showed complete resolution and subsequent gastroscope, echocardiogram and ophthalmological examination were unremarkable. Although liver abscesses caused by Candida albicans rarely occur in immunocompetent patients, it should be included in the differential diagnosis in such patients presenting with suspicious features post laparoscopic cholecystectomy and IOC.

\section{OSTEOMYELITIS CAUSED BY AGGREGATIBACTER APHROPHILUS - A CASE REPORT}

Abhinav Rajkumar $^{1}$, Gayana P. S. Gunaratna ${ }^{1,2}$,

$\overline{\text { Kristine Macartney }}^{1}$, Alexander C. Outhred ${ }^{1}$, Alison Kesson

${ }^{1}$ Department of Infectious Diseases and Microbiology, The Children's Hospital, Westmead, NSW, Australia; and ${ }^{2}$ Faculty of Medicine, University of Kelaniya, Sri Lanka

Osteomyelitis in children is often secondary to haematogenous dissemination of bacteria into long bone metaphyses. Organisms causing osteomyelitis vary with age, and the virulence of different pathogens influences clinical presentation. ${ }^{1}$ Aetiological diagnosis is important in determining appropriate antibiotic therapy.

This case involves an 11-year-old girl with 4 months worsening left knee pain and difficulty weight bearing. She had no fever, preceding trauma or recent dental procedures. Inflammatory markers were unremarkable, with radiological evidence of a Brodie's abscess. She was trialled on oral cephalexin; despite transient clinical improvement, she re-presented with worsening symptoms. 